\title{
A solar filament disconnected by magnetic reconnection ${ }^{\star}$
}

\author{
Zhike Xue ${ }^{1,2,3}$, Xiaoli Yan ${ }^{1,2}$, Liheng Yang ${ }^{1,2}$, Jincheng Wang ${ }^{1,2}$, Qiaoling $\mathrm{Li}^{1,2,4}$, and Li Zhao ${ }^{1,2}$ \\ 1 Yunnan Observatories, Chinese Academy of Sciences, Kunming Yunnan 650216, PR China \\ e-mail: zkxue@ynao.ac.cn \\ 2 Center for Astronomical Mega-Science, Chinese Academy of Sciences, Beijing 100012, PR China \\ 3 CAS Key Laboratory of Solar Activity, National Astronomical Observatories, Beijing 100012, PR China \\ ${ }^{4}$ University of Chinese Academy of Sciences, Beijing 100049, PR China
}

Received 22 October 2019 / Accepted 10 December 2019

\begin{abstract}
Aims. We aim to study a high-resolution observation of an asymmetric inflow magnetic reconnection between a filament and its surrounding magnetic loops in active region NOAA 12436 on 2015 October 23.

Methods. We analyzed the multiband observations of the magnetic reconnection obtained by the New Vacuum Solar Telescope (NVST) and the Solar Dynamic Observatory. We calculated the NVST H $\alpha$ Dopplergrams to determine the Doppler properties of the magnetic reconnection region and the rotation of a jet.

Results. The filament firstly becomes active and then approaches its southwestern surrounding magnetic loops (L1) with a velocity of $9.0 \mathrm{~km} \mathrm{~s}^{-1}$. During this period, the threads of the filament become loose in the reconnection region and then reconnect with L1 in turn. $\mathrm{L} 1$ is pressed backward by the filament with a velocity of $5.5 \mathrm{~km} \mathrm{~s}^{-1}$, and then the magnetic reconnection occurs between them. A set of newly formed loops are separated from the reconnection site with a mean velocity of $127.3 \mathrm{~km} \mathrm{~s}^{-1}$. In the middle stage, some threads of the filament return back first with a velocity of $20.1 \mathrm{~km} \mathrm{~s}^{-1}$, and others return with a velocity of $4.1 \mathrm{~km} \mathrm{~s}^{-1}$ after about $07: 46 \mathrm{UT}$. Then, L1 also begins to return with a velocity of $3.5 \mathrm{~km} \mathrm{~s}^{-1}$ at about 07:47 UT. At the same time, magnetic reconnection continues to occur between them until 07:51 UT. During the reconnection, a linear typical current sheet forms with a length of $5.5 \mathrm{Mm}$ and a width of $1.0 \mathrm{Mm}$, and a lot of hot plasma blobs are observed propagating from the typical current sheet. During the reconnection, the plasma in the reconnection region and the typical current sheet always shows redshifted feature. Furthermore, the material and twist of the filament are injected into the newly longer-formed magnetic loops by the magnetic reconnection, which leads to the formation of a jet, and its rotation.

Conclusions. The observational evidence for the asymmetric inflow magnetic reconnection is investigated. We conclude that the magnetic reconnection does occur in this event and results in the disconnection of the filament. The looseness of the filament may be due to the pressure imbalance between the inside and outside of the filament. The redshifted feature in the reconnection site can be explained by the expansion of the right flank of the filament to the lower atmosphere because of the complex magnetic configuration in this active region.
\end{abstract}

Key words. Sun: filaments, prominences - magnetic reconnection - Sun: activity - Sun: magnetic fields

\section{Introduction}

Magnetic reconnection, a reconfiguration of magnetic lines in the conductive plasma, is an important physical process in which magnetic energy is converted into thermal and kinetic energy. It usually occurs at null points, current sheets, or the quasiseparatrix layers. Magnetic reconnection is generally considered to be associated with solar activities, for example, flares, coronal mass ejections, filament eruptions, and jets (Shibata et al. 1992; Sturrock 1966; Lin \& Forbes 2000; Moore et al. 2001; Yan et al. 2018; Cheng et al. 2018). Magnetic reconnection can cause coronal condensation and helical structure, which may result in the formation of filament or prominence (Kaneko \& Yokoyama 2017; Li et al. 2018, 2019; Xue et al. 2017). In the past decades, lots of observational evidence for magnetic reconnection in the Sun has been reported in many papers, including changes in the connectivity of coronal loops, plasma inflows and outflows, cusp-shaped loops, current-sheet, supra-arcade downflows, shrinking loops, loop-top hard X-ray sources, and so on

\footnotetext{
* Movies associated to Figs. 2-4 are available at https:// www . aanda. org
}

(Asai et al. 2004; Li et al. 2016a; Masuda et al. 1994; Su et al. 2013; Takasao et al. 2012; Tsuneta et al. 1992; Xue et al. 2016, 2018; Yokoyama et al. 2001). Recently, based on high-resolution observation data, several activity events related to small-scale magnetic reconnections have been observed in the lower solar atmosphere (Huang et al. 2018; Tian et al. 2018; Xue et al. 2016, 2017, 2018, 2019; Yan et al. 2016; Yang et al. 2015), while small-scale reconnections are thought to be one candidate for corona heating (e.g., Longcope \& Tarr 2015; Wilmot-Smith 2015). Although magnetic reconnection has been studied in numerous events, many open questions remain mysterious, including: (1) What triggers magnetic reconnection? (2) How does current sheet form? (3) What is the relationship between magnetic reconnection and solar eruptions? Thus, further observations are still needed to understand them.

For the three-dimensional (3D) magnetic reconnection, it often relates to complex magnetic structures. When a filament erupts, it sometimes reconnects with the surrounding magnetic loops, and as a result, the structure of the filament is destroyed and then erupts. Xue et al. (2016) found that a filament reconnects with chromospheric fibrils and then the twist in the filament is released by the magnetic reconnection. Li et al. (2016a) 
reported a successive magnetic reconnection between an erupting filament and its nearly coronal loops, which leads to the changes in the filament connection. Xue et al. (2017) analyzed a tether-cutting reconnection event that results in the formation of a flux rope. While magnetic reconnection is believed to occur in fan-spine configuration where there may be a mini-filament under the null point, and it can reconnect with spine lines and then form a jet (Joshi et al. 2015; Li et al. 2017; Xu et al. 2017). Although magnetic reconnections associated with filaments, including small-scale filaments, are often reported during their eruptions (e.g., Joshi et al. 2018; Yang et al. 2017), the detailed process of magnetic reconnection is rarely noticed in the previous studies.

In this Paper, the high-resolution observation of a smallscale asymmetric inflow magnetic reconnection between a filament and its southwest surrounding magnetic loops is presented using the data obtained by the New Vacuum Solar Telescope (NVST; Liu et al. 2014) and the Solar Dynamic Observatory (SDO; Pesnell 2012) on 2015 October 23. The observations are described in Sect. 2. Section 3 shows the results, while the summary and discussion are presented in Sect. 4.

\section{Data and methods}

The data are mainly the $\mathrm{H} \alpha$ observations of the NVST and extreme ultraviolet (EUV) images and photospheric magnetograms observed by the SDO. The NVST is a ground-based $1 \mathrm{~m}$ vacuum solar telescope in the Fuxian Solar Observatory (FSO) of the Yunnan Observatories, Chinese Academy of Sciences (CAS). It observes the solar photosphere and chromosphere at multiple wavelengths. On 2015 October 23, the $\mathrm{H} \alpha$ images were observed at three wavelength positions, namely the $\mathrm{H} \alpha$ line center, and the line wing at $\pm 0.7 \AA$, with a spatial resolution of $0^{\prime \prime}$.33 and a cadence of $49 \mathrm{~s}$. Furthermore, the Dopplergrams were constructed using the following equation (Langangen et al. 2008),

$D=\frac{B-R}{B+R}$

where $B$ and $R$ are the blue-wing $(\mathrm{H} \alpha-0.7 \AA)$ and red-wing $(\mathrm{H} \alpha+0.7 \AA)$ data, respectively. The EUV images in the $304 \AA$, $171 \AA, 211 \AA, 335 \AA, 131 \AA$, and $94 \AA$ channels obtained by the Atmospheric Imaging Assembly (AIA; Lemen et al. 2012) onboard SDO were employed to show the magnetic reconnection and associated loops at higher temperatures. Additionally, the photospheric magnetograms obtained by the Helioseismic and Magnetic Imager (HMI; Scherrer et al. 2012) and the AIA 304 and $211 \AA$ A running-difference images, which were produced by subtracting the images $24 \mathrm{~s}$ earlier, were used to display the connectivities of the magnetic loops and jet.

\section{Results}

On 2015 October 23, the NVST observed a portion of the active region (AR) NOAA 12436 (Figs. 1a and b) with a $\beta \gamma$ magnetic configuration. In the southwest region close to the leading sunspot with a negative polarity (N1, see Fig. 1f), a small-scale filament is clearly seen in the $\mathrm{H} \alpha$ image (the red dotted line in Fig. 1a) and has an inverse-J-shaped structure. One of its footpoints is rooted in the leading negative polarity (N1), and another one is in a nearby positive polarity (P2). A small-scale magnetic reconnection is observed occurring between this filament and a set of its surrounding magnetic loops (named as L1). A small segment of L1 is observed clearly by the NVST (the blue dotted line in Fig. 1a). Subsequently, it can be seen that the connectivities of the filament and L1 have been changed, and thus two sets of newly magnetic loops (L2 and L3 marked by the pink and white lines in Fig. 1b) are produced by the magnetic reconnection. Since the NVST only observed a small region, there is the problem of where the footpoints of the magnetic loops involved in the magnetic reconnection are. Therefore, we checked the EUV images before the reconnection (Fig. 1e) and found that the signal of the reconnecting loops is too weak to observe them clearly. However, we found that a lot of hot plasma is ejected into the newly formed magnetic loops along two directions during the reconnection in the AIA $304 \AA$ images and $211 \AA$ runningdifference images (the blue dotted line in Figs. 1c and d). One direction is along a path similar to loops from the reconnection site to the east region (close to P1), and another one is along the loops from the reconnection site to N2 (see also Fig. 4 and its attached movie). Thus, most L1 can be traced in the AIA 304 and $211 \AA$ channels. Additionally, another set of magnetic loops (L4) are clearly observed in the $211 \AA$ images, which connect the two main polarities (P1 and N1) of this AR (the green dotted line in Fig. 1e). We did not observe significant brightenings at the possible left footpoint of L1 in either the UV or the EUV images. Even so, based on the similar directions of the most portions of L1 and L4, we conclude that the left footpoint of L1 may be rooted in the same polarity (P1) as the left footpoint of L4. If the positions of the filament, L1 and L4 are overlaid on the HMI magnetogram (Fig. 1f). It is easy to see that the overall magnetic structure may be like the tangent plane of a fan-spine configuration (e.g., Pariat et al. 2009; Török et al. 2009).

Figure 2 displays the detailed process of the small-scale magnetic reconnection in the NVST $\mathrm{H} \alpha$ wavelength (see also the attached movie of Fig. 2). The filament first becomes active at about 07:36 UT, and it begins to expand mainly into a small region marked by the black rectangle in Fig. 2a. To obtain the movement velocity of the filament, a time-distance diagram (Fig. 2j) was made along slice S1 marked by the white dotted line in Fig. 2a, and thus the velocity is estimated to be $9.0 \mathrm{~km} \mathrm{~s}^{-1}$. At first, the filament, as a whole, approaches L1, and then its threads become loose in the inflow region, meaning that the filament becomes wider. However, no similar phenomena are observed in other parts of the filament. We tracked three typical threads and demonstrated their evolution in Figs. 2b-d using the white, pink, and yellow dotted lines, while the other parts of the filament are indicated by the red dotted lines. It is clear that the thread marked by the white line first meets L1, and it is stretched into a linear structure from its original arch. Then, it reconnects with L1, firstly at around 07:43 UT. Then, the other threads approach L1 and reconnect with it in turn. It is also clear that when the L1 is pressed by the filament, it moves slightly backward to the southwest with a velocity of $5.5 \mathrm{~km} \mathrm{~s}^{-1}$ before about 07:47 UT and then returns to the northeast and approaches the filament with a velocity of $3.5 \mathrm{~km} \mathrm{~s}^{-1}$ (the cyan line in Fig. 2j). This is different from the previous observations that magnetic loops move to the reconnection site at the same time (e.g., Su et al. 2013; Xue et al. 2016, 2019). The magnetic reconnection lasts until about 07:51 UT, and then no obvious evidence of the magnetic reconnection is observed. After the reconnection, the filament and L1 exchange their footpoints, leading to the formations of another two sets of magnetic loops (L2 and L3, the white arrows in Fig. 2e). This is also the direct observation evidence of magnetic reconnection. In addition, we would like to point out that some magnetic loops (the pink arrow in Fig. 2f) reappear in the initial position of the filament after the reconnection. 

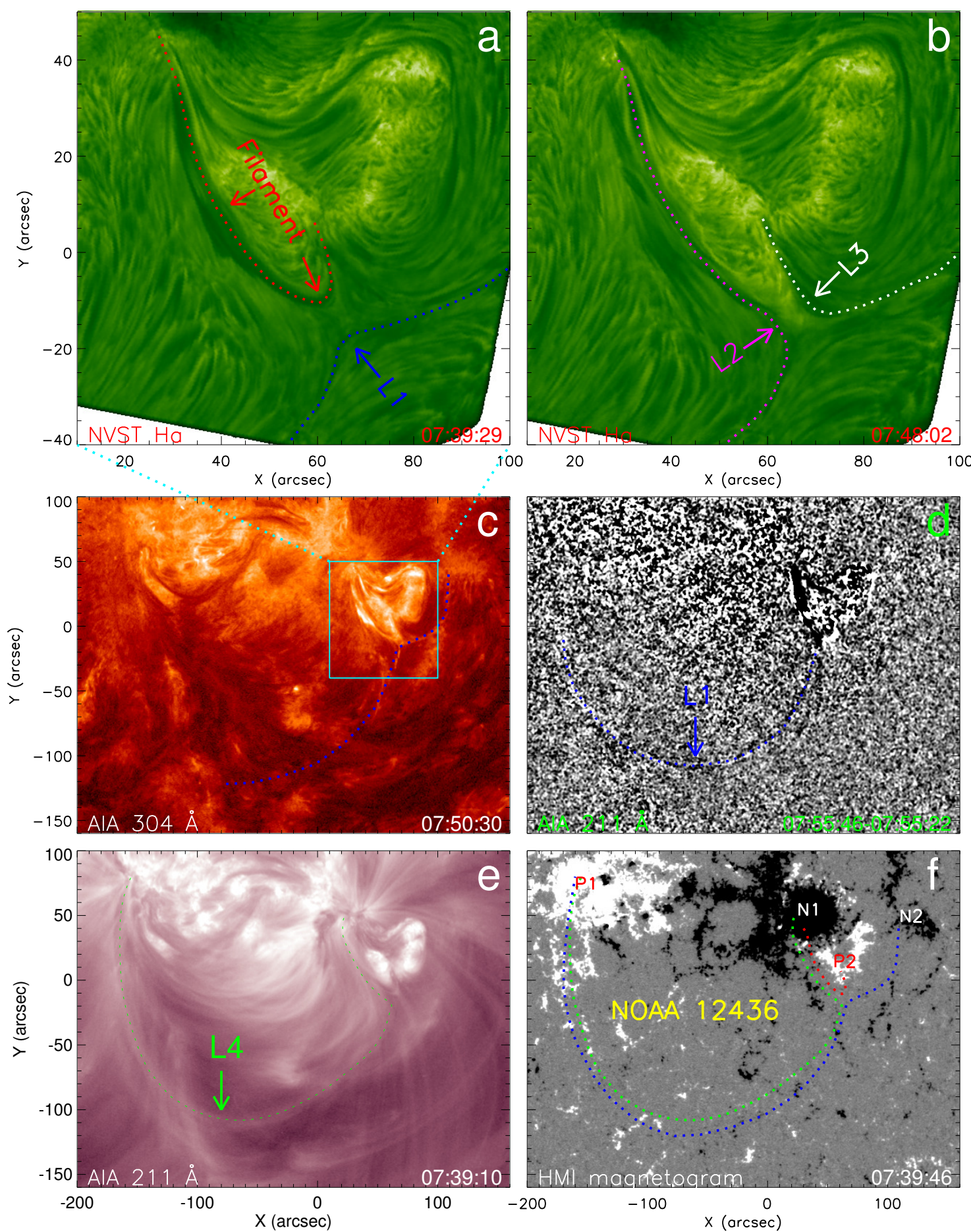

Fig. 1. Overview of magnetic reconnection in the NVST H $\alpha$ (panels $a$ and $b$ ), AIA $304 \AA$, $211 \AA$ running-difference and $211 \AA$ original images (panels $c-e$ ), and HMI magnetogram (panel f). The filament is marked by the red dotted lines and arrows. L1 and L4 are indicated by the blue and green dotted lines and arrows. The newly formed magnetic loops (L2 and L3) after the reconnection are marked by the pink and white dotted lines and arrows. Four magnetic polarities associated with the reconnecting loops are denoted as P1, P2, N1, and N2 (panelf).

The $\mathrm{H} \alpha$ Dopplergrams (Figs. 2g-i and its attached movie) also show the filament eruption and the small-scale magnetic reconnection. At the beginning of the eruption, the left part of the filament shows a blueshifted feature (the blue arrow in Fig. $2 \mathrm{~g}$ ), while the right part has the redshifted feature (the red arrow in Fig. 2g), respectively, indicating that the motions of the two parts have different directions. Similar to the $\mathrm{H} \alpha$ line-center images, the threads of the filament are observed to become loose in the Dopplergrams (the green lines in Fig. 2h), which exhibit redshift before and during the reconnection. In addition, we also observed that some threads do not reconnect with L1, although they move to L1 and then return back again (Fig. 2i), and they also appear as redshifted features. However, the fallback movement is not observed in the $\mathrm{H} \alpha$ line-center images. We conclude that it may be due to the thermal disappearance of the threads heated by the magnetic reconnection, and then they reappear in $\mathrm{H} \alpha$ line-center images (Fig. 2f) caused by the re-cooling after the reconnection (Soru-Escaut \& Mouradian 1990). Additionally, 

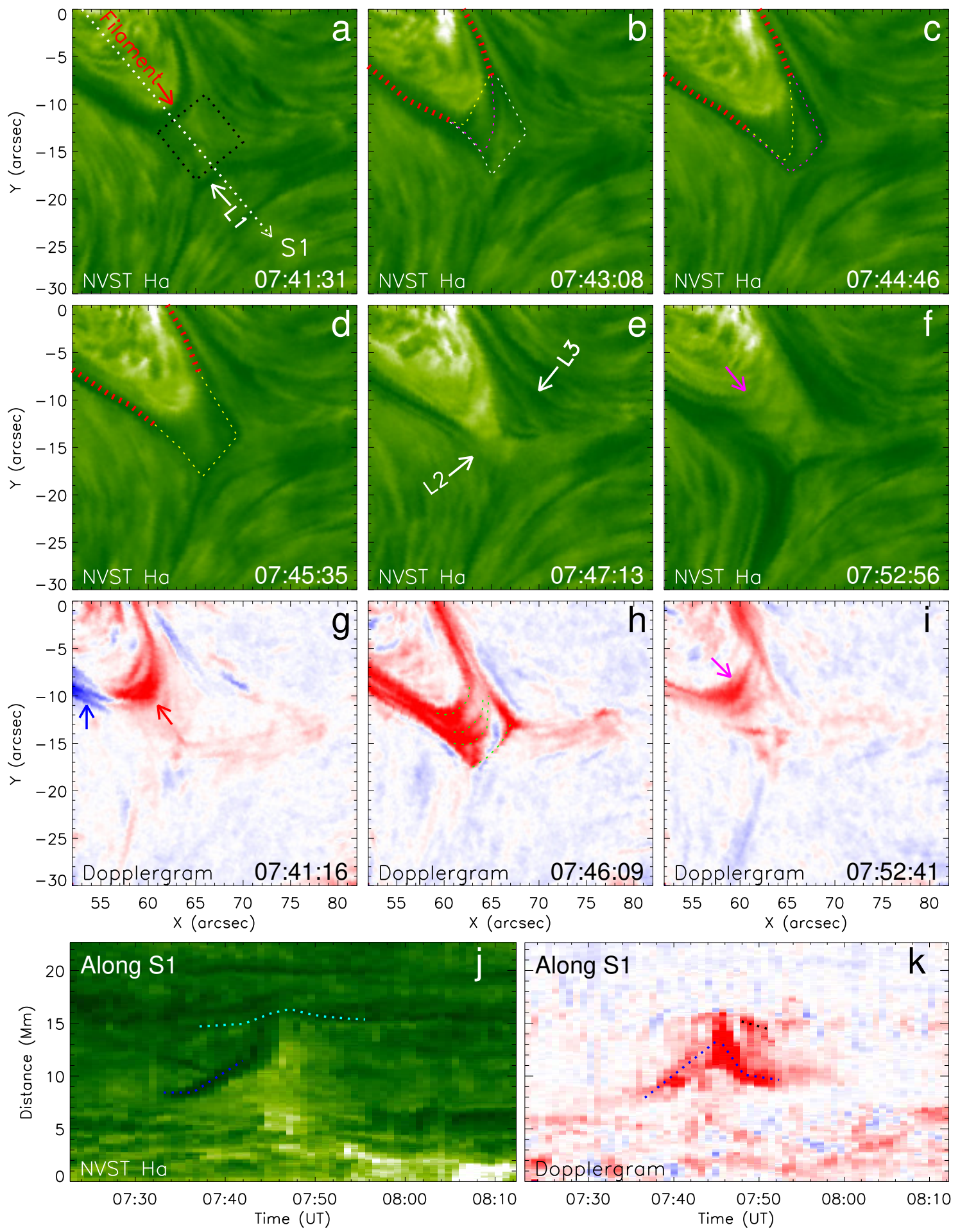

Fig. 2. Evolution of magnetic reconnection in the NVST H $\alpha$ center images (panels a-f) and Dopplergrams (panels g-i). The filament and the magnetic loops (L1) that reconnect in the small region marked by the black rectangle are indicated by the red and white arrows in panel a, respectively. The red, white, pink, yellow and green dotted lines represent the main body and the typical threads of the filament, respectively. The redshifted, blueshifted, and fallback parts of the filament are indicated by the red, blue and pink arrows in panels $f$, $g$, and $i$. The white arrows in panel $e$ point to the newly formed loops (L2 and L3). Panels $j$ and $k$ : the time-distance diagrams obtained along the white dotted line (S1) in panel $a$ using the $\mathrm{H} \alpha$ line-center images and Dopplergrams. The blue, black, and cyan dotted lines mark the motions of the filament and L1. A movie associated to this figure is available online.

the time-distance diagram (Fig. 2k) obtained along S1 using the Dopplergrams shows that the filament moves toward L1 with a velocity of $11.0 \mathrm{~km} \mathrm{~s}^{-1}$. However, after about 07:46 UT, some threads return with a velocity of $20.1 \mathrm{~km} \mathrm{~s}^{-1}$ at first, and then decrease to $2.0 \mathrm{~km} \mathrm{~s}^{-1}$ (the blue lines in Fig. $2 \mathrm{k}$ ), and others return with a velocity of $4.1 \mathrm{~km} \mathrm{~s}^{-1}$ (the black line in Fig. $2 \mathrm{k}$ ). Therefore, it can be seen that the plasma always shows redshift in the reconnection site.

In the AIA EUV channels (Fig. 3 and its attached movie), the magnetic reconnection can also be seen clearly. Besides the 

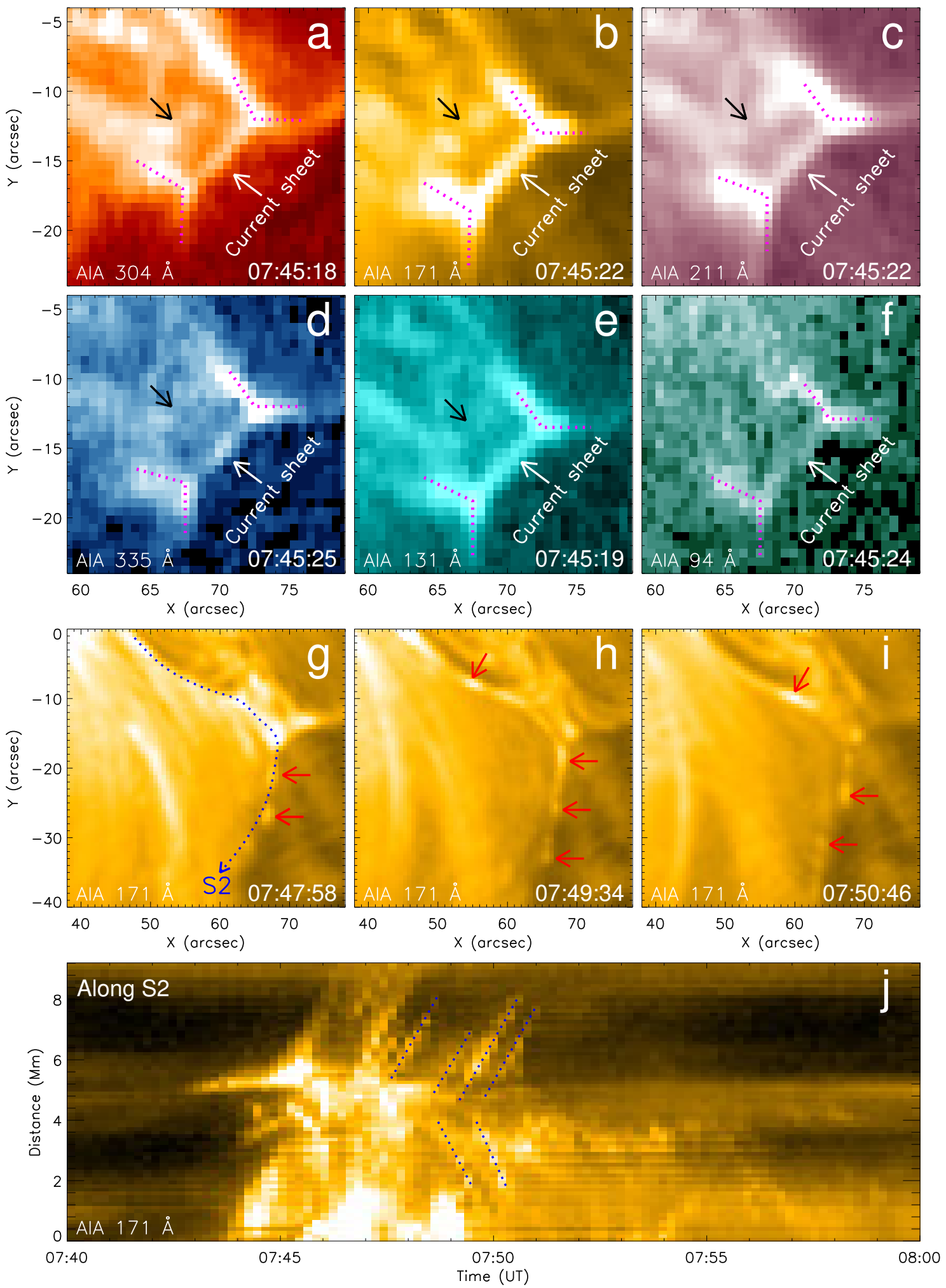

Fig. 3. Magnetic reconnection observed in the EUV channels. The white, black and red arrows refer to the typical current sheet, the currentsheet-liked structure, and the plasma blobs. The hot cusp-shaped structures are indicated by the pink lines. A time-distance diagram (panel $j$ ) was obtained along the blue dotted line (S2) using the AIA $171 \AA$ Amages, in which the motions of the blobs are marked by the blue dotted lines. A movie associated to this figure is available online.

changes of the footpoints of the filament and L1, a typical current sheet can be seen forming at the reconnection site in the multiple channels. It shows a bright linear structure marked by the white arrows in Figs. 3a-f. However, this current sheet is not seen in the $\mathrm{H} \alpha$ channel, but it is clear that the threads of the filament are stretched into a linear structure at the reconnection site (see Fig. 2). This indicates that this current sheet has a higher temperature. Additionally, the length and width of the typical 
current sheet are estimated to be about $5.5 \mathrm{Mm}$ and $1.0 \mathrm{Mm}$, which results in a reconnection rate of 0.18 , similar to the previous results (e.g., Xue et al. 2016, 2018). At the same time, two hot cusp-shaped structures (the pink lines in Figs. 3a-f) are observed at the two ends of the typical current sheet. Additionally, a current-sheet-like structure is observed in the northeastern region of the reconnection site near the typical current sheet in the EUV images except $94 \AA$ images at around 07:45 UT, which also appears as a bright linear structure (the black arrows in Figs. 3a-e). Besides, multiple current-sheet-like structures can be seen in the AIA $304 \AA$ images at around 07:50 UT (see the attached movie of Fig. 4), but are not observed in other AIA EUV channels. The temperature of the current-sheet-like structures may be lower than that of the typical current sheet. We conclude that the current-sheet-like structure may be the heated threads of the filament by the magnetic reconnection as mentioned above, or may be a real current sheet. If the latter is true, it implies that multiple current sheets are observed at the same time during the magnetic reconnection, which may be caused by the magnetic reconnections between the loose threads of the filament and different fine structures of L1, sequentially. Therefore, magnetic reconnection may be a complex process in 3D. In previous studies, multiple current sheet structures were discovered during one erupting event (e.g. Li et al. 2016b).

Furthermore, during the reconnection, a lot of plasma blobs are observed only in the EUV channels, but have no signal in the $\mathrm{H} \alpha$ images. They are seen most clearly in the $171 \AA$ images from 07:47 UT to 07:51 UT, which are indicated by the red arrows in Figs. $3 g-i$. The plasma blobs move along two sets of the magnetic loops from the southeast end of the typical current sheet to the footpoints $\mathrm{N} 1$ and $\mathrm{P} 1$. When the plasma blobs come out from the typical current sheet, they are the brightest, then their signals become gradually weaker and weaker and finally disappear, and they do not spread to the footpoints $\mathrm{N} 1$ and P1. To show the kinematics of these plasma blobs, a time-distance diagram (Fig. 3j) was calculated along the slice S2 based on the AIA $171 \AA$ images in which the typical blobs are indicated by the blue dotted lines. By fitting their motions with the linear functions, the velocities of the blobs are estimated to be from $37.7 \mathrm{~km} \mathrm{~s}^{-1}$ to $51.3 \mathrm{~km} \mathrm{~s}^{-1}$ with an average velocity of $43.1 \mathrm{~km} \mathrm{~s}^{-1}$.

During the reconnection, many magnetic loops (L2, marked by the white arrows in Figs. $4 \mathrm{a}$ and $\mathrm{b}$ ) are observed to form at the left outflow region. They are composed of a part of the filament threads and L1, and their footpoints are rooted in the polarities $\mathrm{P} 1$ and N1. They gradually move away from the reconnection site with the velocities from $98.2 \mathrm{~km} \mathrm{~s}^{-1}$ to $140.3 \mathrm{~km} \mathrm{~s}^{-1}$, which are obtained from the time-distance diagram (Fig. 4l) along the slice S3 indicated by the white line in Fig. 4a. Thus, their average velocity is estimated to be $127.3 \mathrm{~km} \mathrm{~s}^{-1}$. Subsequently, the material in the left part of the filament is injected into the left portion of L1 and propagates along them, leading to the formation of a jet (the green arrows in Figs. $4 \mathrm{c}-\mathrm{g}$ ) whose plasma is from the filament resulting from the reconnection. The jet can be seen clearly in the running-difference images. In the AIA $304 \AA$ running-difference images (Figs. $4 c-d$ ), we found that when the jet propagates to the middle part of L2, it gradually becomes invisible. However, in the AIA $211 \AA$ running-difference images (see Figs. $4 \mathrm{e}-\mathrm{g}$ and its attached movie), the jet moves farther to the left leg of L2 near polarity P1. The time-distance diagram along the slice S4 (the green line in Fig. 4e) shows the propagation of the jet, and its velocity is calculated to be $283.4 \mathrm{~km} \mathrm{~s}^{-1}$ (see Fig. 4m). The jet does not propagate to the polarity $\mathrm{P} 1$ to cause remote brightenings as shown in previous studies (e.g., Wang \& Liu 2012; Xu et al. 2017). Additionally, we found that the jet rotates counterclockwise if viewed from the top to the footpoint N1 (see the attached movie of Fig. 4), indicating that there may be a flux rope that may be related to the filament before the reconnection (e.g., Joshi et al. 2018). Furthermore, the rotation of the jet is also shown in the H $\alpha$ Dopplergrams (Figs. 4h-k and the attached movie of Fig. 2). A blueshift at the initial stage in Fig. 4h indicates that the magnetic structure rises as a whole. Then, the Dopplergrams (Figs. $4 \mathrm{i}$ and $\mathrm{j}$ ) show that the east edge of the jet has a redshifted feature (the blue arrows) while the west edge is blueshifted (the black arrows). This means that the plasma on both sides of the jet moves in the opposite direction. In the time-distance diagram (Fig. 4n) obtained along the slice S5 (the pink line in Fig. 4h), it is easy to find that the plasma always exhibits redshift (the green line) in the left part of the jet, while it always shows blueshift (the yellow line) in the right part within its duration. This reveals that the blueshift at one edge and redshift at another edge have been observed in many events, and are believed to be a typical signature of the rotational motion of a jet (e.g., Pike \& Mason 1998; Curdt \& Tian 2011; Wang et al. 2018). Additionally, we noted that not all of the filament material propagates along the left portion of L2 in the form of the jet, some of which fall back to the footpoint N1 (marked by the purple arrow in Fig. 4k).

\section{Summary and discussion}

Using the high-resolution data obtained by the NVST and SDO, we studied the small-scale magnetic reconnection between the filament and the magnetic loops in the AR NOAA 12436 on 2015 October 23 . The typical observational evidence is observed including the exchanges of the footpoints of the filament and its southwestern surrounding magnetic loops, the reconnection inflows and outflows, the newly formed magnetic loops, the linear typical current sheet with a length of $5.5 \mathrm{Mm}$ and a width of $1.0 \mathrm{Mm}$, two hot cusp-shaped structures, and the movement of many blobs from the reconnection site with an average velocity of $43.1 \mathrm{~km} \mathrm{~s}^{-1}$.

In this event, when the filament approaches L1 with a velocity of $9.0 \mathrm{~km} \mathrm{~s}^{-1}, \mathrm{~L} 1$ is not seen to move toward the filament at the same time. In contrast, L1 begins to move backward to the southwest, with a velocity of $5.5 \mathrm{~km} \mathrm{~s}^{-1}$. It can be explained as the erupting filament meets L1 leading to the pile up of the field lines, and thus L1 is pressed toward the southwest by the erupting filament. Subsequently, the filament reconnects with L1. Here, we find that the movement directions of the inflow plasma on both sides of the typical current sheet are the same, from northeast to southwest, in the beginning. Then, some threads of the filament are seen to return first with a velocity of $20.1 \mathrm{~km} \mathrm{~s}^{-1}$, and others return with a velocity of $4.1 \mathrm{~km} \mathrm{~s}^{-1}$ after about 07:46 UT, and then L1 also begins to return with a velocity of $3.5 \mathrm{~km} \mathrm{~s}^{-1}$ at about 07:47 UT. In this stage, the directions of the movements of the filament and L1 are also the same (from southwest to northeast), and the velocities of L1 and the slowmoving threads of the filament are similar. Therefore, the reconnection continues until about 07:51 UT. In the standard magnetic reconnection models, the plasma velocities in two inflow regions are almost the same and their movement directions are reversed (e.g., Parker 1957; Petschek 1964; Sweet et al. 1958) However, in observations, the inflows on either side of null-point or current sheet have different velocities (e.g., Su et al. 2013; Xue et al. 2016), and these magnetic reconnections are called as asymmetric inflow magnetic reconnection. They are observed to 

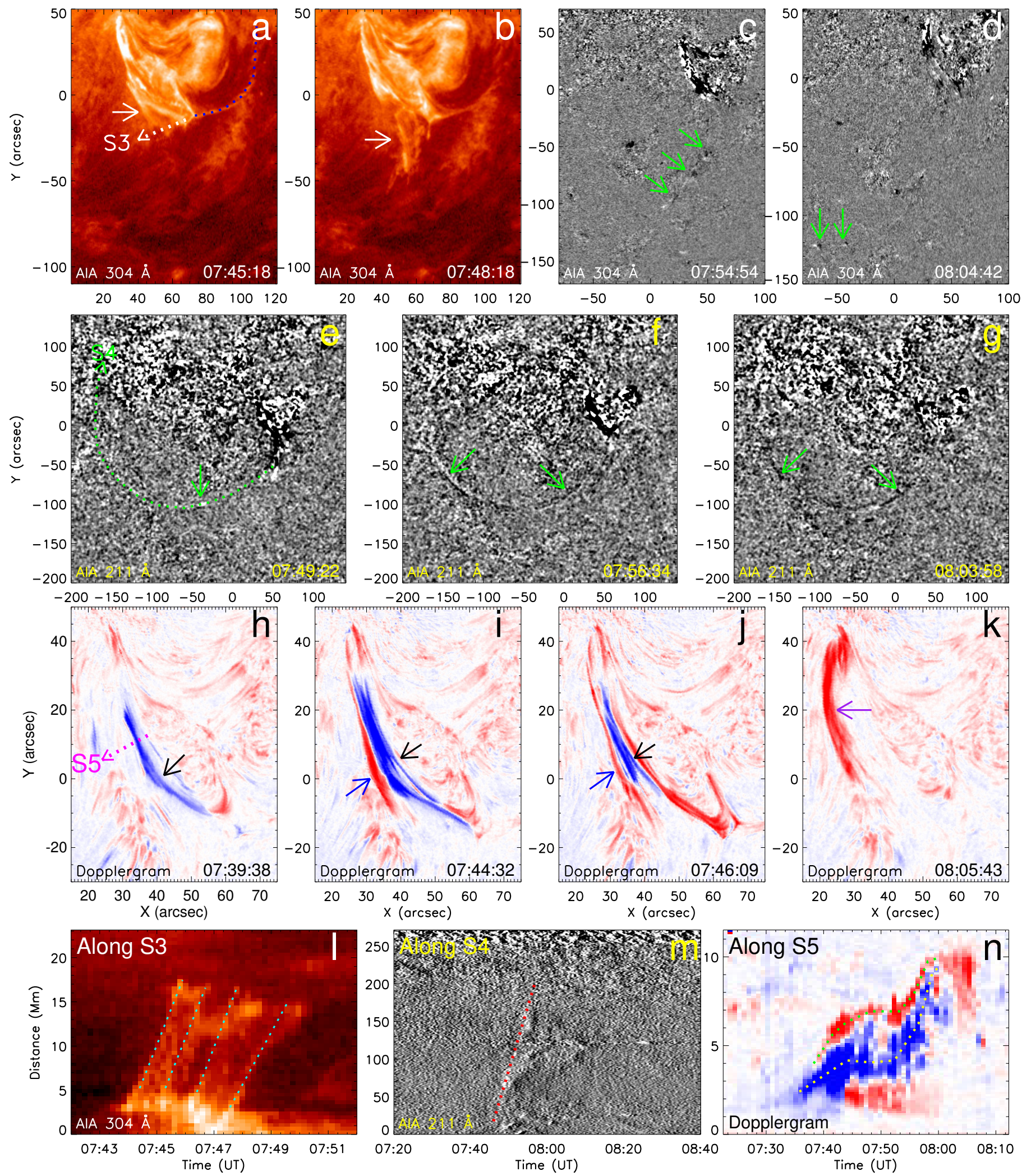

Fig. 4. Jet shown in the AIA $304 \AA$ original (panels $a$ and $b$ ) and AIA $304 \AA$ running-difference images (panels $c$ and $d$ ), AIA $211 \AA$ runningdifference images (panels $e-g$ ), and in the $\mathrm{H} \alpha$ Dopplergrams (panels $h-k$ ). Three time-distance diagrams (panels $l-n$ ) were obtained along the slice S3 (the white line in panel a) using the AIA $304 \AA \AA$ images, along the slice S4 (the green line in panel e) using the AIA 211 A running-difference images, and along the slice S5 (the pink line in the panel $h$ ) using the $\mathrm{H} \alpha$ Dopplergrams. The white arrows point to the newly formed loops. The jet is indicated by the green arrows, and its redshifted and blueshifted portions are marked by the blue and black arrows in panels $h-j$ and green and yellow lines in panel $n$, respectively. The motions of the newly formed loops are indicated by the cyan lines in panel $l$, and the jet in panel $m$ is indicated by the red line. The purple arrow points to the downward filament material. A movie associated to this figure is available online. 
occur in Earth's magnetopause, laboratory plasma experiments, and plasma turbulence. The asymmetric inflow magnetic reconnection may be triggered by the different magnetic field strengths and plasma densities in each inflow region (Murphy et al. 2012; Murphy \& Lukin 2015). In the lower solar atmosphere, it is often thought that the asymmetric inflow magnetic reconnection occurs between newly emerged flux and preexisting, overlying flux (e.g., Shibata et al. 1992; Moore et al. 2013), while the observation of asymmetric inflow magnetic reconnection involving filament has been seldom reported.

Another interesting observational phenomenon is that the threads of the filament become loose at the reconnection site before the magnetic reconnection, and thus the filament becomes wider. Then, the loose threads gradually reconnect with L1. While in the rest of the filament, we did not observe a clear loose structure before the reconnection. This may indicate that the filament swells at the reconnection site. The expansion of the main body of the dark strip tends to be observed when the dark strip erupts into the higher Sun's atmosphere. However, the expansion of filament body is often observed when the filament erupts to the higher atmosphere, and this may be due to the pressure imbalance between the inside and outside of the filament (e.g., Chen 2011; Gibson 2018). Similarly, in this event, the pressure at the reconnection site may be smaller than that inside the filament. Therefore, due to the pressure difference, the movement velocity of the threads closest to the reconnection site is the fastest, and that of the threads farther away from the reconnection site is the slowest, and this may result in the loosening of the filament at the reconnection site.

Furthermore, the plasma near the reconnection site, including that in the typical current sheet, always shows a redshifted feature before and during the magnetic reconnection. We conclude that this observation may be due to the magnetic topology. The filament erupts firstly, and its right flank extends into the lower atmosphere. Then the filament presses L1 to the lower right and reconnects with it. In this process, both the magnetic structures and the plasma move away from the observer. Therefore, the redshifted feature can be seen in the Dopplergrams (Fig. 2). This indicates that the magnetic reconnection site may be lower than the apex of the filament. On the contrary, Xue et al. (2018) found that current sheet shows blueshift at the middle and redshift at the ends, and concluded that these results are likely to be related to the motion of the magnetic structures or to the turbulences in the current sheet.

Acknowledgements. We gratefully acknowledge insightful and constructive comments and suggestions by the anonymous referee which led to significant improvements of this paper. We thank the NVST and SDO teams for providing high-resolution data. This work is sponsored by the National Science Foundation of China (NSFC) under the grant numbers 11973084, 11873087, 11803085, 11803086, 11633008, Yunnan Science Foundation of China under numbers 2018FB007 and 2018FA001, Youth Innovation Promotion Association, CAS (Nos 2019061, 2011056), CAS "Light of West China" Program, the grant associated with the Project of the Group for Innovation of Yunnan Province and CAS Key Laboratory of Solar Activity, National Astronomical
Observatories under number KLSA201910. X.L.Y. thanks ISSI-BJ for supporting him to attend the team meeting led by J. C. Vial and P. F. Chen.

\section{References}

Asai, A., Yokoyama, T., Shimojo, M., \& Shibata, K. 2004, ApJ, 605, L77 Chen, P. F. 2011, Liv. Rev. Sol. Phys., 8, 1

Cheng, X., Li, Y., Wan, L. F., et al. 2018, ApJ, 866, 64

Curdt, W., \& Tian, H. 2011, A\&A, 532, L9

Gibson, S. E. 2018, Liv. Rev. Sol. Phys., 15, 7

Huang, Z. H., Mou, C. Z., Fu, H., et al. 2018, ApJ, 853, L26

Joshi, N. C., Liu, C., Sun, X., et al. 2015, ApJ, 812, 50

Joshi, N. C., Nishizuka, N., Filippov, B., Magara, T., \& Tlatov, A. G. 2018, MNRAS, 476, 1286

Kaneko, T., \& Yokoyama, T. 2017, ApJ, 845, 12

Langangen, Ø., Rouppe van der Voort, L., \& Lin, Y. 2008, ApJ, 673, 1201

Lemen, J. R., Title, A. M., Akin, D. J., et al. 2012, Sol. Phys., 275, 17

Li, L. P., Zhang, J., Hardi, P., et al. 2016a, Nat. Phys., 12, 847

Li, L. P., Zhang, J., Su, J. T., \& Liu, Y. 2016b, ApJ, 829, L33

Li, H. D., Jiang, Y. C., Yang, J. Y., et al. 2017, ApJ, 842, L20

Li, L. P., Zhang, J., Peter, H., et al. 2018, ApJ, 864, L4

Li, L. P., Peter, H., Chitta, L. P., et al. 2019, ApJ, 884, 34

Lin, J., \& Forbes, T. G. 2000, J. Geophys. Res., 105, 2375

Liu, Z., Xu, J., Gu, B. Z., et al. 2014, Res. Astron. Astrophys., 14, 705

Longcope, D. W., \& Tarr, L. A. 2015, Phil. Trans. R. Soc. London Ser. A, 373 , 20140263

Masuda, S., Kosugi, T., Hara, H., Tsuneta, S., \& Ogawara, Y. 1994, Nature, 371, 495

Moore, R. L., Sterling, A. C., Hudson, H. S., \& Lemen, J. R. 2001, ApJ, 552, 833

Moore, R. L., Sterling, A. C., Falconer, D. A., \& Robe, D. 2013, ApJ, 769, 134 Murphy, N. A., \& Lukin, V. S. 2015, ApJ, 805, 134

Murphy, N. A., Miralles, M. P., Pope, C. L., et al. 2012, ApJ, 751, 56

Parker, E. N. 1957, J. Geophys. Res., 62, 509

Pariat, E., Antiochos, S. K., \& DeVore, C. R. 2009, ApJ, 691, 61

Pesnell, W. D., Thomopson, B. J., \& Chamberlin, P. C., 2012, Sol. Phys., 275, 3

Petschek, H. E. 1964, NASA Spec. Pub., 50, 425

Pike, C. D., \& Mason, H. E. 1998, Sol. Phys., 182, 333

Scherrer, P. H., Schou, J., Bush, R. I., et al. 2012, Sol. Phys., 275, 207

Shibata, K., Ishido, Y., Acton, L. W., et al. 1992, PASJ, 44, L173

Soru-Escaut, I., \& Mouradian, Z. 1990, Sol. Phys., 230, 474

Sturrock, P. A. 1966, Nature, 211, 695

Su, Y., Veronig, A. M., Holman, G. D., et al. 2013, Nat. Phys., 9, 489

Sweet, P. A. 1958, in Electromagnetic Phenomena in Cosmical Physics, ed.

B. Lehnert (Cambridge: Cambridge Univ. Press), IAU Symp., 6, 123

Takasao, S., Asai, A., Isobe, H., \& Shibata, K. 2012, ApJ, 745, L6

Tian, H., Zhu, X. S., Peter, H., et al. 2018, ApJ, 854, 174

Török, T., Aulanier, G., Schmieder, B., Reeves, K. K., \& Golub, L. 2009, ApJ, 704, 485

Tsuneta, S., Ishido, Y., Acton, L. W., et al. 1992, PASJ, 44, L63

Wang, H., \& Liu, C. 2012, ApJ, 760, 101

Wang, J. C., Yan, X. L., Qu, Z. Q., et al. 2018, ApJ, 863, 180

Wilmot-Smith, A. L. 2015, Phil. Trans. R. Soc. London Ser. A, 373, 20140265

Xu, Z., Yang, K., Guo, Y., et al. 2017, ApJ, 851, 30

Xue, Z. K., Yan, X. L., Cheng, X., et al. 2016, Nat. Commun., 7, 11837

Xue, Z. K., Yan, X. L., Yang, L. H., Wang, J. C., \& Zhao, L. 2017, ApJ, 840, L23

Xue, Z. K., Yan, X. L., Yang, L. H., et al. 2018, ApJ, 858, L4

Xue, Z. K., Yan, X. L., Jin, C. L., et al. 2019, ApJ, 874, L27

Yan, X. L., Priest, E. R., Guo, Q. L., et al. 2016, ApJ, 832, 23

Yan, X. L., Yang, L. H., Xue, Z. K., et al. 2018, ApJ, 853, L18

Yang, S. H., Zhang, J., \& Xiang, Y. Y. 2015, ApJ, 798, L11

Yang, L. H., Yan, X. L., Li, T., Xue, Z. K., \& Xiang, Y. Y. 2017, ApJ, 838, 131

Yokoyama, T., Akita, K., Morimoto, T., Inoue, K., \& Newmark, J. 2001, ApJ, 546, L69 